\title{
Steady-State Security Control Using a Sensitivity-Based Approach
}

\author{
CHUNG-LIANG CHANG* and YUAN-YIH HSU \\ Department of Electrical Engineering, National Taiwan University, Taipei (Taiwan) \\ (Received May 30, 1989)
}

\section{ABSTRACT}

Real time security assessment is gaining more and more attention with the increasing complexity of electrical power systems. Steadystate security control is made to keep the system in a state without violating any constraints. In this paper, an algorithm based on the firstorder sensitivities is presented both for real power and for reactive power security control. Relations between uncompensated sensitivity factors used for corrective control and compensated sensitivity factors used for preventive control have been derived theoretically. It is found that the combined effects of a line outage and a line switching cannot be summed directly, because of inherent nonlinearity. The effectiveness of the proposed method is investigated by study. ing the Taiwan power system.

\section{INTRODUCTION}

With the growing size of power systems and the complexity of operating conditions, power system security, which deals with the system's ability to withstand outage events, is of greater concern to the system operator as well as to the system planner than it was in the past. In order to assess the security of a power system, a proper classification of security levels is necessary. A security regime can be divided into normal, alert, emergency and restorative states $[1,2]$. For the purpose of steady-state security, the alert state indicates that the system is operating within limits, but a contingency analysis program predicts there will be an MW overload or a voltage violation

\footnotetext{
*Present address: System Planning Department, Taiwan Power Company, 242 Roosevelt Road, Section 3, Taipei 10763, Taiwan
}

problem when a certain outage occurs. Preventive control should be taken to improve security, that is, to return the system to the normal state. In an emergency state, the system is in danger of overloads or voltage violations unless corrective control is taken to restore it at least to the alert state.

Steady-state security control strategies are categorized according to whether they primarily affect real power or reactive power problems. Actions that can be taken to relieve power flow overloads include the control of phase shifter flows, area interchanges, generation rescheduling, load shifting and line switching, etc. In the event of voltage problems, actions that can be taken are transformer tap adjustments, generator voltage adjustments and capacitor/reactor switching, etc. In the literature, numerous approaches have been devised to realize the above control actions. Some of these utilized the optimization techniques such as the optimal power flow (OPF) [3-5]. There are no new requirements for use of OPF for security control, except for additional constraints imposed on the limits of the circuit loadings, bus voltages, and control actions, etc. For the security-constrained OPF using the linear programming approach, it is necessary to calculate the sensitivities of each constraint with respect to the controlling parameters in order to get the linearized operating constraints.

There are some different opinions about the optimal solution of the security problem. When abnormalities occur in a system due to a contingency, optimal dispatch is not, in general, of greatest concern to the operator, but rather system security.

Hence, some other methods stressing the ability to make recommended decisions to the dispatcher to enhance the security are proposed, with no attempt to find the optimal solution. A typical approach is the network 
sensitivity method [6]. There are two types of sensitivities: generation shift factors with respect to generation rescheduling, and line outage distribution factors with respect to line switching. Extending generation shift factors to include load shift factors is straightforward if loads are allowed to be adjusted. These factors are not required to be precomputed but can be obtained efficiently by using the sparse matrix inverse technique $[7,8]$. The control actions determined by the network sensitivity method or optimal power flow must be identified through an AC load flow test [8].

The concept of sensitivity factors has been used extensively in various power system problems. The network sensitivity method, based on the DC load flow, is not suitable for voltage correction. Although use of the linearized reactive power model alone may not work well for the purpose of reactive power contingency selection, it is still worthwhile to see whether this approach is suitable for voltage correction. Based on the linearized reactive power flow equations, the sensitivity factors relating the voltage changes to the changes of voltage correction actions can be defined as in the real power case. The results shown in this paper reveal that this approach is rather promising. It is useful to know that not all of the sensitivity factors of the system are required; only the terms related to the overloaded branches or voltage violated buses are calculated [8]. These terms are calculated using relevant elements of the sparse impedance and branch reactances.

By combining the effects of contingency outages and the changes of control actions simultaneously, the modified (or compensated) sensitivity factors can be used to achieve the preventive control. In other words, should a certain outage cause a violation problem, the same corrections can be made using compensated factors as were run with the uncompensated factors. In general, the combined effects can be obtained using superposition. However, it is shown in this paper that, unless certain conditions are satisfied, the combined effects of a line outage and a line switching cannot be added directly because of their nonlinear relationships.

The main features of this paper are summarized as follows: (1) the method based on the linearized AC power flow is proposed for eliminating system overloads and voltage viola- tions; (2) relations between the sensitivity factors, compensated sensitivity factors, corrective controls and preventive controls are carefully examined; (3) steady-state security control of a real power system (Taiwan power system) is analyzed using the proposed method.

\section{METHODOLOGY OF REAL POWER CONTROL}

\subsection{Linearized AC power flow}

The linearized AC power flow can be obtained simply from the fast decoupled load flow model [9] by setting the $V$ terms of the forcing function to 1.0 per unit:

$\mathbf{B}^{\prime} \Delta \boldsymbol{\theta}=\Delta \boldsymbol{P}$

$\mathbf{B}^{\prime \prime} \Delta V=\Delta \boldsymbol{Q}$

where $\Delta \boldsymbol{P}$ and $\Delta \boldsymbol{Q}$ are vectors of real and reactive power changes, $\Delta \theta$ and $\Delta V$ vectors of changes in voltage angles and magnitudes,

$B_{i j}^{\prime}=-1 / x_{i j}, \quad B_{i i}^{\prime}=\sum\left(1 / x_{i j}\right)$

$B_{i j}^{\prime \prime}=b_{i j}, \quad B_{i i}^{\prime \prime}=-\sum b_{i j}-\mathrm{SH}_{i}$

$x_{i j}$ and $b_{i j}$ are the reactance and susceptance of branch $i-j$, and $\mathrm{SH}_{i}$ is the total shunt admittance at bus $i$.

Both matrices $\mathbf{B}^{\prime}$ and $\mathbf{B}^{\prime \prime}$ are real, symmetrical and sparse but in different dimensions since the swing bus is excluded from $\mathbf{B}^{\prime}$ and $P V$ buses are excluded from $\mathbf{B}^{\prime \prime}$. Using the technique mentioned in ref. 10 , both matrices can be triangularized using the same ordering, regardless of bus types. Equations (1) and (2) can be rewritten as

$\Delta \theta=\mathbf{X}^{\prime} \Delta P$

$\Delta \boldsymbol{V}=\mathrm{X}^{\prime \prime} \Delta \boldsymbol{Q}$

where $\mathbf{X}^{\prime}$ and $\mathbf{X}^{\prime \prime}$ are the sparse inverses of $\boldsymbol{B}^{\prime}$ and $\mathrm{B}^{\prime \prime}$, respectively.

Since Taiwan power system is the only power system in Taiwan and there are no phase shifters, the actions being considered to alleviate overloads are generation adjustments, load shifts and line switching.

The methods for reducing overloads are discussed in this section, while the voltage problem is left to the next section.

\subsection{Generation adjustment and load shift}

2.2.1. Corrective control

Assume that overloaded branch $l$ is to be relieved. The effect of adjusting generations 
or loads on the flow of branch $l$ can be evaluated with generation/load shift factors:

$A(l, i)=\frac{1}{x_{l}}\left(X_{n i}^{\prime}-X_{m i}^{\prime}\right)$

where $X_{n i}^{\prime}$ and $X_{m i}^{\prime}$ are elements of $\mathbf{X}^{\prime}, x_{l}$ is the reactance of line $l, n$ and $m$ are terminal buses of line $l$, and $i$ is an adjustable generator or load bus.

Total effects of simultaneous changes on several buses can be calculated using superposition. Thus, the reduction of flow on branch $l$ requires

$$
\begin{aligned}
\Delta P_{l}= & P_{l}-\bar{P}_{l} \leqslant \sum_{i \in \Omega_{\mathrm{G}}}\left|A(l, i) \Delta P_{i}\right| \\
& +\sum_{j \in \Omega_{\mathrm{L}}}\left|A(l, j) \Delta P_{j}\right|
\end{aligned}
$$

where $\bar{P}_{l}$ is the power limit of line $l, P_{l}$ the base-case flow in line $l$ directed from $n$ to $m$, $\Omega_{\mathrm{G}}$ the set of adjustable generator buses, $\boldsymbol{\Omega}_{\mathrm{L}}$ the set of adjustable load buses, $\Delta P_{i}$ the change in injected power at generator bus $i$, and $\Delta P_{j}$ the change in injected power at load bus $j$.

The direction of the adjustments can be decided according to the sign of the shift factor, namely,

- increasing generation at bus $i$ if $A(l, i)<0$

- decreasing generation at bus $i$ if $A(l, i)>0$

- increasing load at bus $j$ if $A(l, j)>0$

- decreasing load at bus $j$ if $A(l, j)<0$

The amount of adjustment at generator bus $i$ is the minimum $\left(\Delta P_{i}^{\mathrm{c}}\right)$ of values between the generation limit at bus $i$, the generation limit at the reference bus and $\left|\Delta P_{i}\right| A(l, i) \mid$. The amount at load bus $j$ is the minimum of values between the load change limit and $\left|\Delta P_{j}\right| A(l, j) \mid$.

The next step is to determine which buses should be included in sets $\Omega_{\mathrm{G}}$ and $\Omega_{\mathrm{L}}$. Clearly, $\Omega_{\mathrm{L}}$ will be an empty set if load shifts are avoided, and generation rescheduling is executed prior to load shifting. A bus in sets $\Omega_{\mathrm{G}}$ or $\Omega_{\mathrm{L}}$ must satisfy the following requirements: (1) the bus with a higher value of $\left|A(l, i) \Delta P_{i}^{\mathrm{c}}\right|$ is put into the sets prior to the bus with a lower one, and (2) it must not cause overload of any other branch. Following the requirements, buses are entered into the sets $\Omega_{\mathrm{G}}$ or $\Omega_{\mathrm{L}}$ until eqn. (6) is satisfied.
The flow diagram of generation rescheduling including load shifting is shown in Fig. 1.

\subsubsection{Preventive control}

Should a line outage at line $k$ occur, the generation/load shift factor between overloaded branch $l$ and bus $i$ is

$\hat{A}(l, i)=\frac{1}{x_{l}}\left(\hat{X}_{n i}^{\prime}-\hat{X}_{m i}^{\prime}\right)$

where the symbol ${ }^{\wedge}$ represents the outaged condition.

Elements $\hat{X}_{n i}^{\prime}$ and $\hat{X}_{m i}^{\prime}$ can be replaced by the elements $X_{n i}^{\prime}$ and $X_{m i}^{\prime}$ of the base case using the matrix inversion lemma:

$\hat{X}_{n i}^{\prime}=X_{n i}^{\prime}+\frac{\left(X_{p n}^{\prime}-X_{q n}^{\prime}\right)\left(X_{p i}^{\prime}-X_{q i}^{\prime}\right)}{x_{k}-\left(X_{p p}^{\prime}+X_{q q}^{\prime}-2 X_{p q}^{\prime}\right)}$

$\hat{X}_{m i}^{\prime}=X_{m i}^{\prime}+\frac{\left(X_{p m}^{\prime}-X_{q m}^{\prime}\right)\left(X_{p i}^{\prime}-X_{q i}^{\prime}\right)}{x_{k}-\left(X_{p p}^{\prime}+X_{q q}^{\prime}-2 X_{p q}^{\prime}\right)}$

where $x_{k}$ is the reactance of line $k$, and $p$ and $q$ are the terminal buses of line $k$.

So,

$$
\begin{aligned}
\hat{A}(l, i)= & \frac{1}{x_{k}}\left(X_{n i}^{\prime}-X_{m i}^{\prime}\right) \\
& +\frac{x_{k}}{x_{l}} \frac{\left(X_{p n}^{\prime}-X_{q n}^{\prime}\right)-\left(X_{p m}^{\prime}-X_{q m}^{\prime}\right)}{x_{k}-\left(X_{p p}^{\prime}+X_{q q}^{\prime}-2 X_{p q}^{\prime}\right)} \\
& \times \frac{1}{x_{k}}\left(X_{p i}^{\prime}-X_{q i}^{\prime}\right) \\
= & A(l, i)+D(l, k) A(k, i)
\end{aligned}
$$

where $D(l, k)$ is the line outage distribution factor between lines $l$ and $k$ in a base case. The right-hand side of eqn. (10) is the so-called compensated generation/load shift factor. Since compensated factors are calculated us ing the $X^{\prime}$ matrix of the base case, they can be used for preventive control to alleviate violations due to a contingency.

Equation (11a) reveals that the combined effects of a line outage and generation/load shifting control can be obtained using superposition. That is to say,

$$
\begin{aligned}
\Delta P_{l} & =[A(l, i)+D(l, k) A(k, i)] \Delta P_{i} \\
& =A(l, i) \Delta P_{i}+D(l, k) A(k, i) \Delta P_{i} \\
& =A(l, i) \Delta P_{i}+D(l, k) \Delta P_{k}
\end{aligned}
$$




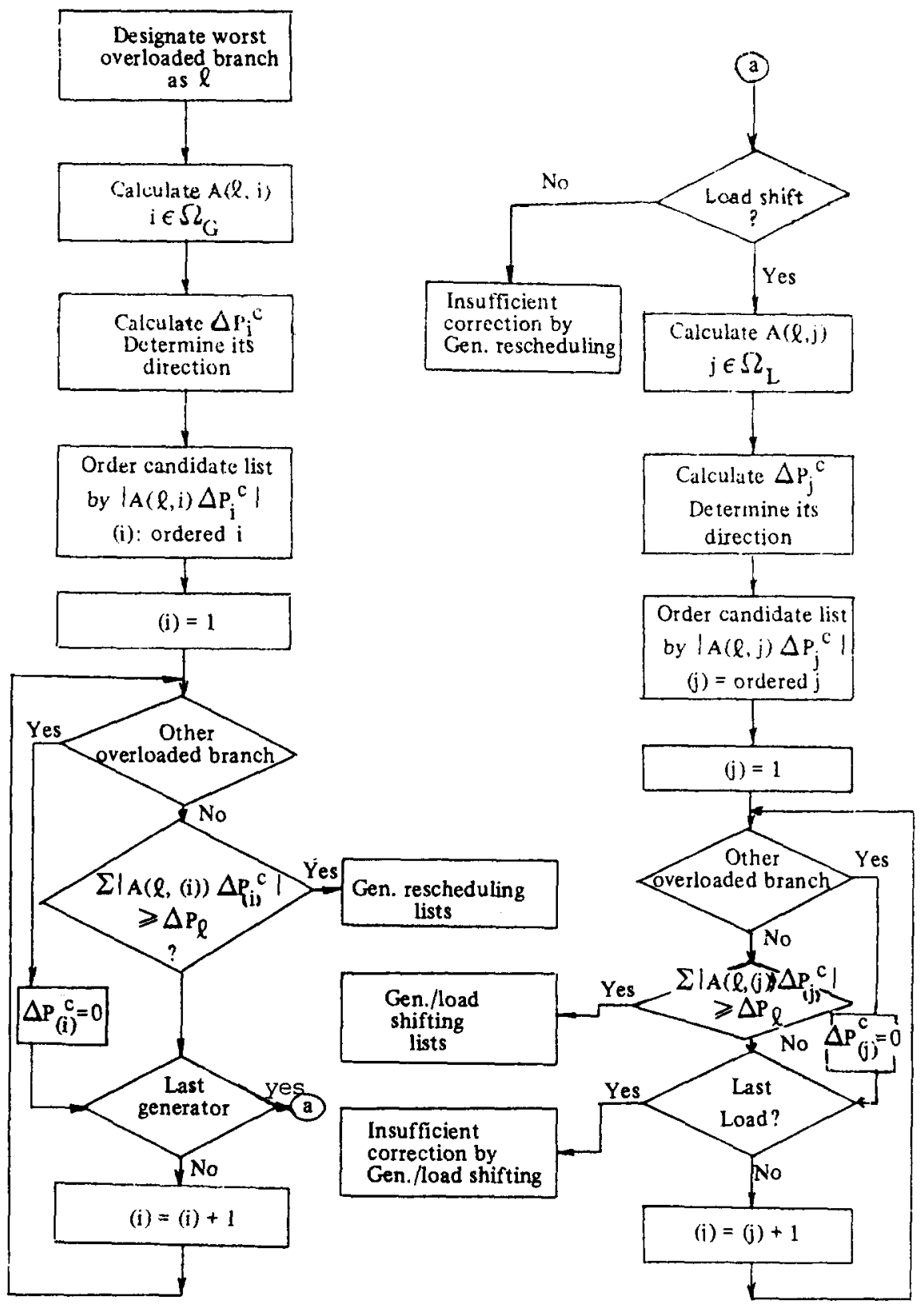

Fig. 1. Generation/load shifting algorithm.

\subsection{Line switching}

\subsubsection{Corrective control}

The line outage distribution factor between overloaded branch $l$ and dropping branch $t$ is

$D(l, t)=\frac{x_{t}}{x_{l}} \frac{\left.X_{r n}^{\prime}-X_{s n}^{\prime}\right)-\left(X_{r m}^{\prime}-X_{s m}^{\prime}\right)}{x_{t}-\left(X_{r r}^{\prime}+X_{s s}^{\prime}-2 X_{r s}^{\prime}\right)}$

where $x_{t}$ is the reactance of line $t$, and $r$ and $s$ are the terminal buses of line $t$.

Only the lines corresponding to negative distribution factors can be selected for switching. The algorithm for switching is shown in Fig. 2.

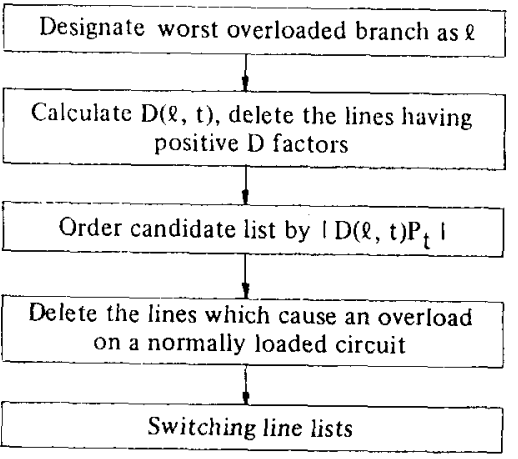

Fig. 2. Line switching algorithm. 


\subsubsection{Preventive control}

Should a line outage at line $k$ occur, the distribution factor between overloaded branch $l$ and switching line $t(k \neq t)$ is

$\hat{D}(l, t)=\frac{x_{t}}{x_{l}} \frac{\left(\hat{X}_{r n}^{\prime}-\hat{X}_{s n}^{\prime}\right)-\left(\hat{X}_{r m}^{\prime}-\hat{X}_{s m}^{\prime}\right)}{x_{t}-\left(\hat{X}_{r r}^{\prime}+\hat{X}_{s s}^{\prime}-2 \hat{X}_{r s}^{\prime}\right)}$

Substitution of the 'capped' values with 'uncapped' values gives

$$
\begin{aligned}
\hat{D}(l, t)= & \frac{x_{t}}{x_{l}}\left\{\left[x_{k}-\left(X_{p p}^{\prime}+X_{q q}^{\prime}-2 X_{p q}^{\prime}\right)\right]\right. \\
& \times\left[\left(X_{r n}^{\prime}-X_{s n}^{\prime}\right)-\left(X_{r m}^{\prime}-X_{s m}^{\prime}\right)\right] \\
& +\left[\left(X_{p n}^{\prime}-X_{q n}^{\prime}\right)-\left(X_{p m}^{\prime}-X_{q m}^{\prime}\right)\right] \\
& \left.\times\left[\left(X_{p r}^{\prime}-X_{q r}^{\prime}\right)-\left(X_{p s}^{\prime}-X_{q s}^{\prime}\right)\right]\right\} \\
& \times\left\{\left[x_{k}-\left(X_{p p}^{\prime}+X_{q q}^{\prime}-2 X_{p q}^{\prime}\right)\right]\right. \\
& \times\left[x_{t}-\left(X_{r r}^{\prime}+X_{s s}^{\prime}-2 X_{r s}^{\prime}\right)\right] \\
& \left.-\left[\left(X_{p r}^{\prime}-X_{q r}^{\prime}\right)-\left(X_{p s}^{\prime}-X_{q s}^{\prime}\right)\right]^{2}\right\}^{-1}
\end{aligned}
$$

If

$$
\begin{aligned}
{\left[x_{k}\right.} & \left.-\left(X_{p p}^{\prime}+X_{q q}^{\prime}-2 X_{p q}^{\prime}\right)\right] \\
& \times\left[x_{t}-X_{r r}^{\prime}-\left(X_{r r}^{\prime}-X_{s s}^{\prime}-2 X_{r s}^{\prime}\right)\right] \\
& \gg\left[\left(X_{p r}^{\prime}-X_{q r}^{\prime}\right)-\left(X_{p s}^{\prime}-X_{q s}^{\prime}\right)\right]^{2}
\end{aligned}
$$

then $\hat{D}(l, t)$ can be rewritten as

$$
\begin{aligned}
\hat{D}(l, t)= & \frac{x_{t}}{x_{l}} \frac{\left(X_{r n}^{\prime}-X_{s n}^{\prime}\right)-\left(X_{r m}^{\prime}-X_{s m}^{\prime}\right)}{x_{t}-\left(X_{r r}^{\prime}+X_{s s}^{\prime}-2 X_{r s}^{\prime}\right)} \\
& +\frac{x_{k}}{x_{l}} \frac{\left(X_{p n}^{\prime}-X_{q n}^{\prime}\right)-\left(X_{p m}^{\prime}-X_{q m}^{\prime}\right)}{x_{k}-\left(X_{p p}^{\prime}+X_{q q}^{\prime}-2 X_{p q}^{\prime}\right)} \\
& \times \frac{x_{t}}{x_{k}} \frac{\left(X_{r p}^{\prime}-X_{s p}^{\prime}\right)-\left(X_{r q}^{\prime}-X_{s q}^{\prime}\right)}{x_{t}-\left(X_{r r}^{\prime}+X_{s s}^{\prime}-2 X_{r s}^{\prime}\right)} \\
= & D(l, t)+D(l, k) D(k, t)
\end{aligned}
$$

Only under the condition of eqn. (15) can the combined effects of a line outage and a line switching be added directly, or

$\Delta P_{l}=D(l, t) \Delta P_{t}+D(l, k) \Delta P_{k}$

However, the condition of eqn. (15) may not be satisfied for all possible switching lines. This is equivalent to saying that, in general, the effect on the power flow of tripping a line with another circuit out of service (as in the corrective case) and that of tripping two lines subsequently in the normal case (as in the preventive case) are not equal.

\section{METHODOLOGY OF REACTIVE POWER CONTROL}

In this section, the method of voltage control based on the linearized reactive power equations is considered. Control actions under consideration include generator voltage adjustment, transformer tap adjustment and capacitor/reactor switching. Although the tap and capacitor/reactor are discrete in nature, the analysis will be performed using the continuous approach. Thus, the closest integer to the computed value is used for discrete setting. In the rest of this section, the voltage at bus $j$ is assumed to be relieved.

\subsection{Generator remote voltage adjustment}

3.1.1. Corrective control

The voltage change at $P V$ bus $i$ creates incremental reactive power injections, $b_{u i} \Delta V_{i}$, at each bus $u$ connected to bus $i$. Using eqn. (4), the sensitivity factor between bus voltage $V_{j}$ and bus voltage $V_{i}$ is

$S_{V V}(j, i)=\frac{\Delta V_{j}}{\Delta V_{i}}=\sum_{u} X_{j u}^{\prime \prime} b_{u i}$

The direction of adjustment is easily decided according to the sign of the factor $S_{V V}$, and the control effort is the minimum of values between the voltage limit and $\left|\Delta V_{j} / S_{V V}(j, i)\right|$, where $\Delta V_{j}$ is the desired amount of correction. The voltage limit at $P V$ bus $i$ can be obtained either from experience or by comparing its $Q$ limit and the reactive generation change, where

$\Delta Q_{i}=V_{i} \sum b_{i u} \Delta V_{u}+V_{i} \Delta V_{i} b_{i i}$

The flow diagram of generator voltage adjustment is shown in Fig. 3.

\subsubsection{Preventive control}

In order to derive the compensated factors $S_{V V}$ for preventive control, two types of sensitivity factor are defined first. Sensitivity factor $S_{J V}(k, i)$ refers to the change in reactive flow in line $k$ with a change in bus voltage $V_{i}$, and sensitivity factor $S_{V J}(j, k)$ refers to the change in bus voltage $V_{j}$ with the base-case reactive flow in line $k\left(J_{k}\right)$. It is shown in the Appendix that

$S_{J V}(k, i)=\sum_{u}\left(X_{p u}^{\prime \prime}-X_{q u}^{\prime \prime}\right) b_{i u}$ 


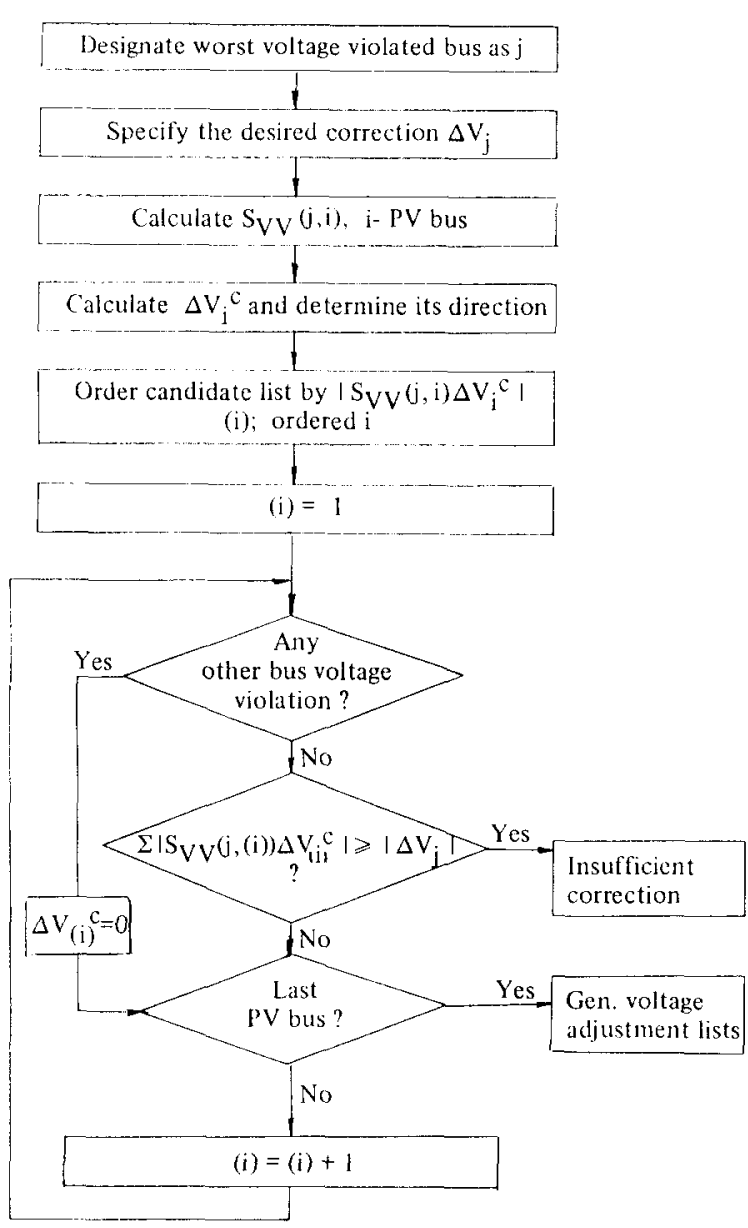

Fig. 3. The algorithm for generator voltage adjustment.

and

$S_{V J}(j, k)=\frac{X_{j p}^{\prime \prime}-X_{j q}^{\prime \prime}}{1-b_{p q}\left(X_{p p}^{\prime \prime}+X_{q q}^{\prime \prime}-2 X_{p q}^{\prime \prime}\right)}$

Following these relations, the compensated factor $\hat{S}_{V V}(j, i)$ for the contingency of a line $k$ outage can be expressed as

$\hat{S}_{V V}(j, i)=S_{V V}(j, i)+S_{V J}(j, k) S_{J V}(k, i)$

Equation (22) reveals that the combined effect of this type of sensitivity factor can be calculated using superposition.

Figure 3 can be used directly for preventive control by replacing factor $S_{V V}$ with factor $\hat{S}_{V V}$

\subsection{Transformer tap adjustment}

\subsubsection{Corrective control}

Let the tap of a transformer in line $l$, having terminal buses $n$ and $m$, be used for correcting the voltage at bus $j$. The tap increment, $\Delta t_{l}$, creates incremental reactive power injections $-\Delta t_{l} b_{n m}$ and $\Delta t_{l} b_{n m}$ at the $n$ and $m$ buses, respectively. Then, by eqn. (4), the sensitivity factor relating a change in bus voltage $\Delta V$, to a change in the tap of a transformer in line $l, \Delta t_{l}$, can be calculated as

$S_{V T}(j, l)=\frac{\Delta V_{j}}{\Delta t_{l}}=\left(X_{j n}^{\prime \prime}-X_{j m}^{\prime \prime}\right) b_{n m}$

The algorithm in Fig. 3 can also be used for tap adjustment with slight modifications.

\subsubsection{Preventive control}

It is easy to show that

$\hat{S}_{V T}(j, l)=S_{V T}(j, l)+S_{V J}(j, k) S_{J T}(k, l)$

where line $k$ is the outaged line, and the sensitivity factor $S_{J T}(k, l)$ refers to the change of reactive flow in line $k$ with a change of transformer tap at branch $l$.

\subsection{Capacitor/reactor (shunt device) switching} 3.3.1. Corrective control

From eqn. (4), the sensitivity factor relating the change in bus voltage $V_{j}$ to a change of shunt admittance at bus $i$ is

$S_{V S}(j, i)=\frac{\Delta V_{j}}{\Delta b_{j}}=X_{j i}^{\prime \prime}$

Figure 3 can still be applied for shunt device switching with slight modifications.

\subsubsection{Preventive control}

It is easy to show that

$\hat{S}_{V S}(j, i)=S_{V S}(j, i)+S_{V J}(j, k) S_{J S}(k, i)$

where line $k$ represents the line outage contingency and the sensitivity factor $S_{J S}(k, i)$ refers to the change of reactive flow in line $k$ with a change of shunt admittance at bus $i$ (see Appendix).

\section{EXAMPLES AND RESULTS}

\subsection{Description of the study system}

The system under study is the Taiwan power (Taipower) system which consists of 23 generators, 195 buses and 251 branches. Of the 251 branches, 97 were transformers with inphase taps. There are no phase shifters in the system. Most of the generators are geographically located in the northern and southern areas of Taiwan. The heavy lines in the oneline diagram depicted in Fig. 4 indicate the 


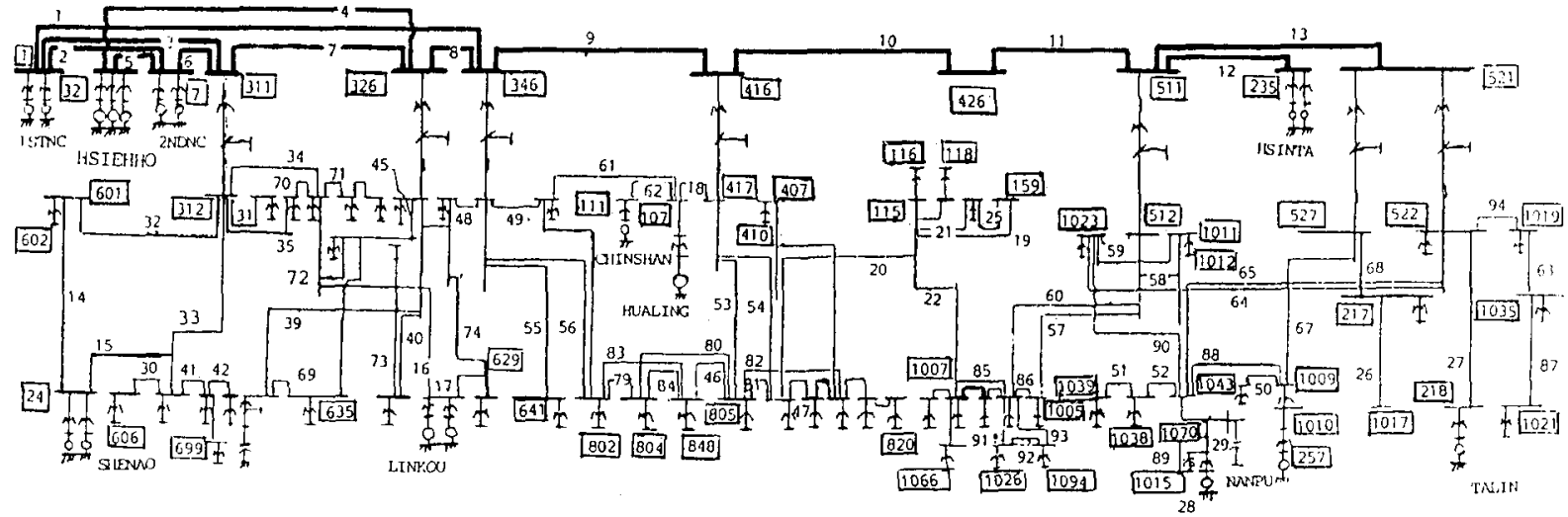

Fig. 4. One-line diagram of Taiwan power system.

$345 \mathrm{kV}$ trunk lines, and the remaining lines are the $161 \mathrm{kV}$ lines. The load level of the base case with all constraints satisfied is $7920.7 \mathrm{MW}$. In the following discussions, steady-state security control will be performed for the contingencies which cause either system overloads or voltage violations. The contingencies which were selected using the contingency selection algorithm are shown in Table 1.

\subsection{Real power security control}

There are two contingencies to be considered, one with a branch which is $102 \%$ loaded and the other with two branches which are $108.7 \%$ and $120.5 \%$ loaded, respectively. Various control actions determined by the sensitivity-based approach are tried to alleviate the

\section{TABLE 1}

Study cases

(a) Contingencies having overload problems

\begin{tabular}{cc}
\hline Outaged branch & Violations (MW\%) \\
\hline $1009-1069$ & $416 \cdot 419(102 \%)$ \\
$115-1001$ & $511-514(120.5 \%)$ \\
& $1009 \cdot 1069(108.7 \%)$
\end{tabular}

(b) Contingencies having voltage problems

\begin{tabular}{cc}
\hline Outaged branch & Violations $\left(\mathrm{V}_{\mathrm{pu}}\right)$ \\
\hline $28-629$ & $630(0.9389), 629(0.9399)$ \\
$346-416$ & $623(0.9492)$ \\
& $803(0.9353), 819(0.9355)$ \\
& $412(0.9357), 809(0.9391)$ \\
& $827(0.9395), 407(0.9396)$ \\
& $805(0.9400), 801(0.9421)$ \\
& $416(0.9428), 807(0.9436)$ \\
& $1065(0.9482), 810(0.9498)$ \\
\end{tabular}

TABLE 2

Generation/load shift factors

(a) Outaged branch: $1009-1069$

Generation shift factors

\begin{tabular}{rlrl}
\hline Bus No. & Value & Bus No. & Value \\
\hline 112 & -0.5950 & 10 & -0.0365 \\
199 & -0.5950 & 33 & -0.0364 \\
30 & -0.0702 & 34 & -0.0364 \\
29 & -0.0702 & 35 & -0.0364 \\
26 & -0.0426 & 3 & -0.0354 \\
9 & -0.0365 & 4 & -0.0354 \\
\hline
\end{tabular}

Load shift factors

\begin{tabular}{llll}
\hline Bus No. & Value & Bus No. & Value \\
\hline 814 & -0.5950 & 410 & -0.5575 \\
808 & -0.5652 & 806 & -0.5524 \\
820 & -0.5575 & 828 & -0.4405 \\
810 & -0.5575 & 848 & -0.4063 \\
415 & -0.5575 & 804 & -0.3880 \\
\hline
\end{tabular}

(b) Outaged branch: 115 - 1001

Generation shift factors

\begin{tabular}{llll}
\hline Bus No. & Value & Bus No. & Value \\
\hline 223 & -0.1605 & 221 & -0.1496 \\
220 & -0.1496 & 222 & -0.1496 \\
\hline
\end{tabular}

Load shift factors

\begin{tabular}{llll}
\hline Bus No. & Value & Bus No. & Value \\
\hline 1002 & -0.7089 & 1040 & -0.2063 \\
1004 & -0.7089 & 1069 & -0.2063 \\
1006 & -0.7089 & 1070 & -0.2063 \\
1008 & -0.7089 & 525 & -0.1605 \\
1012 & -0.7089 & 1020 & -0.1605 \\
1026 & -0.7089 & 1022 & -0.1605 \\
1028 & -0.7089 & 1036 & -0.1605 \\
1066 & -0.7089 & 1010 & -0.1586 \\
1094 & -0.7089 & 1044 & -0.1586 \\
1024 & -0.2376 & 1018 & -0.1496 \\
\hline
\end{tabular}


TABLE 3

Comparison between exact and approximate distribution factors

(a) Outaged branch: $1009 \cdot 1069$

\begin{tabular}{lll}
\hline Dropping branch & Exact value & Approximate value \\
\hline $115-1001$ & -0.8042 & -0.8015 \\
$107-625$ & -0.5832 & -0.5832 \\
$347-349$ & -0.4619 & -0.4619 \\
$346-416$ & -0.2906 & -0.2906 \\
$417-807$ & -0.1993 & -0.1993 \\
\hline
\end{tabular}

(b) Outaged branch: $115-1001$

\begin{tabular}{lll}
\hline Dropping branch & Exact value & Approximate value \\
\hline $512-1023$ & -1.0000 & -0.9494 \\
$522-524$ & -0.3649 & -0.3601 \\
$527-529$ & -0.2020 & -0.2013 \\
$1023-1069$ & -0.1725 & -0.1718 \\
$522-1019$ & -0.0019 & -0.0020 \\
\hline
\end{tabular}

overloads. The sensitivity factors corresponding to the generation shift, load shift and line switching controls are listed in Tables 2 and 3 . From Table 2, it can be seen that, with generation rescheduling only, the case having slight overloading can be corrected completely but the severe case fails, unless load shifting is permitted. The validity of the corrections can be proved by testing an $\mathrm{AC}$ power flow, the results of which are shown in Table 7. From Table 3, it seems that it is possible to use line switching for alleviating overloads. But in fact it cannot be accepted even for the less severe case, because line switching is always accompanied by voltage violations, as can be seen in Table 7 .

It has been mentioned in $\$ 2.3 .2$ that the compensated distribution factors defined in eqn. (10) are not equal to the exact distribu tion factors used for preventive control. However, the results shown in Table 3 reveal that there is no significant difference for those switching lines having higher values of the distribution factors.

\subsection{Reactive power security control}

Two contingencies, one with voltage violations at three buses and the other with voltage violations at twelve buses, are considered. Sensitivity factors corresponding to the generator remote voltage control, transformer tap adjustment and capacitor/reactor switching are shown in Tables 4,5 and 6 , respectively.
TABLE 4

Generator voltage sensitivity factors

(a) Outaged branch: 28 - 629

\begin{tabular}{rccc}
\hline$P V$ bus No. & Value & $P V$ bus No. & Value \\
\hline 9 & 0.1291 & 35 & 0.0677 \\
10 & 0.1291 & 112 & 0.0450 \\
3 & 0.1026 & 237 & 0.0445 \\
4 & 0.1026 & 238 & 0.0443 \\
33 & 0.0677 & 30 & 0.0293 \\
34 & 0.0677 & 223 & 0.0287 \\
\hline
\end{tabular}

(b) Outaged branch: $346-416$

\begin{tabular}{rrrr}
\hline$P V$ bus No. & Value & $P V$ bus No. & Value \\
\hline 9 & 0.09057 & 238 & 0.06899 \\
10 & 0.09057 & 30 & 0.06129 \\
112 & 0.07850 & 29 & 0.05051 \\
3 & 0.07046 & 199 & 0.04918 \\
4 & 0.07046 & 33 & 0.04743 \\
237 & 0.06928 & 34 & 0.04743 \\
\hline
\end{tabular}

TABLE 5

Transformer tap sensitivity factors

(a) Outaged branch: $28-629$

\begin{tabular}{lrlr}
\hline $\begin{array}{l}\text { Transformer } \\
\text { circuit }\end{array}$ & Value & $\begin{array}{l}\text { Transformer } \\
\text { circuit }\end{array}$ & Value \\
\hline $629 \cdot 630$ & 1.0000 & $311-314$ & -0.0329 \\
$346 \cdot 349$ & 0.8193 & $813-814$ & -0.0282 \\
$416 \cdot 417$ & 0.0571 & $521 \cdot 524$ & -0.0224 \\
$326 \cdot 329$ & -0.0446 & $521-529$ & -0.0207 \\
\hline
\end{tabular}

(b) Outaged branch: $346-416$

\begin{tabular}{lrll}
\hline $\begin{array}{l}\text { Transformer } \\
\text { circuit }\end{array}$ & Value & $\begin{array}{l}\text { Transformer } \\
\text { circuit }\end{array}$ & Value \\
\hline $346 \cdot 349$ & 0.3818 & $521-524$ & -0.0312 \\
$416 \cdot 417$ & 0.2720 & $521-529$ & -0.0298 \\
$813-814$ & -0.0478 & $326-329$ & -0.0091 \\
$511-512$ & 0.0461 & $311-314$ & -0.0001 \\
\hline
\end{tabular}

\section{TABLE 6}

Capacitor/reactor sensitivity factors

(a) Outaged branch: 28.629

\begin{tabular}{llll}
\hline Bus No. & Value & Bus No. & Value \\
\hline 630 & 0.0582 & 802 & 0.0094 \\
624 & 0.0231 & 804 & 0.0079 \\
626 & 0.0104 & 808 & 0.0076 \\
\hline
\end{tabular}

(b) Outaged branch: $346-416$

\begin{tabular}{llll}
\hline Bus No. & Value & Bus No. & Value \\
\hline 804 & 0.0278 & 810 & 0.0121 \\
848 & 0.0230 & 415 & 0.0121 \\
802 & 0.0164 & 410 & 0.0121 \\
806 & 0.0130 & 820 & 0.0121 \\
\hline
\end{tabular}




\section{TABLE 7}

Validity of control actions

(a) Overload problems

\begin{tabular}{llll}
\hline $\begin{array}{l}\text { Outaged } \\
\text { branch }\end{array}$ & $\begin{array}{l}\text { Generation } \\
\text { rescheduling }\end{array}$ & $\begin{array}{l}\text { Gen./load } \\
\text { shift }\end{array}$ & $\begin{array}{l}\text { Line } \\
\text { switching }\end{array}$ \\
\hline $1009-1069$ & O.K. & $\begin{array}{l}\text { Not necessary } \\
\text { O.K. }\end{array}$ & $\begin{array}{l}\text { Divergent } \\
\text { Divergent }\end{array}$ \\
\hline $115-1001$ & O.K. & O.K.
\end{tabular}

(b) Voltage problems

\begin{tabular}{rlll}
\hline $\begin{array}{l}\text { Outaged } \\
\text { branch }\end{array}$ & $\begin{array}{l}\text { Gen. voltage } \\
\text { control }\end{array}$ & $\begin{array}{l}\text { Transformer } \\
\text { tap control }\end{array}$ & $\begin{array}{l}\text { Capacitor/ } \\
\text { reactor } \\
\text { switching }\end{array}$ \\
\hline $28 \cdot 629$ & O.K. & O.K. & O.K. \\
$346 \cdot 416$ & O.K. & O.K. & O.K. \\
\hline
\end{tabular}

As can be seen in Table 7, these control actions are all valid for alleviating violations of two contingencies. Hence, the approach based on the linearized reactive power flow equations is suitable for voltage correction.

\section{CONCLUSIONS}

A fast algorithm for steady-state security control is presented in this paper. The algorithm, based on sensitivity analysis, has the ability to handle both overload and voltage problems. The control actions under consideration include generation rescheduling, load shifting, line switching, generator voltage adjustment, transformer tap adjustment and capacitor/reactor switching. It can be concluded from the study of Taiwan power system that the corrections made by the proposed method are successful for eliminating system abnormalities.

Compensated sensitivity factors, used for preventive control, with respect to various control actions are carefully examined in this study. It is found that the combined effects of a line outage contingency and a line switching correction cannot simply be added by superposition.

\section{REFERENCES}

1 T. E. Dy Liacco, The adaptive reliability control system, IEEE Trans., PAS-86 (1967) 517 - 531.

2 T. C. Cihlar, J. H. Wear, D. N. Ewart and L. K.
Kirchmayer, Electric utility system security, Proc. Am. Power Conf., 31 (1969) $891 \cdot 908$.

$3 \mathrm{G}$. Schnyder and H. Glavitsch, Integrated security control using an optimal power flow and switching concepts, IEEE Trans., PWRS.3 (1988) 782 - 790.

4 A. P. Sakis Meliopoulos, R. R. Kovacs, N. D. Reppen, G. Contaxis and N. Balu, Power system remedial action methodology, IEEE Trans., PWRS-3 (1988) $500 \cdot 509$.

5 B. Stott, O. Alsac and A. J. Monticelli, Security analysis and optimization, Proc. IEEE, 75 (1987) 1623 - 1644

6 A. J. Wood and B. F. Wollenberg, Power Generation, Operation and Control, Wiley, New York, 1983.

7 J. E. Fagan, The formation of a sparse bus impedance matrix, Master Thesis, Univ. Texas at Arlington, 1973

8 A. A. Mazi, B. F. Wollenberg and M. H. Hesse, Corrective control of power system flows by line and bus-bar switching, IEEE Trans., PWRS-1 (1986) 258-265.

9 B. Stott and O. Alsac, Fast decoupled load flow, IEEE Trans., PAS.93 (1974) 859 - 867.

$10 \mathrm{~S}$. M. Chan and V. Brandwajn, Partial matrix refactorization, Proc. 14th PICA Conf., 1985, IEEE, New York, pp. 9 - 15 .

\section{APPENDIX}

Derivation of $S_{J V}(k, i)$

By definition

$S_{J V}(k, i) \triangleq \frac{\Delta J_{k}}{\Delta V_{i}}$

where $J_{k}$ is the reactive flow in line $k$ and $V_{i}$ is the voltage at bus $i$. Based on the linearized model and assuming the tap ratios in a base are 1.0 per unit, the incremental reactive flow $\Delta J_{k}$ can be calculated as

$\Delta J_{k}=\left(\Delta V_{p}-\Delta V_{q}\right) b_{p q}$

where $p$ and $q$ are the terminal buses of line $k$, and $b_{p q}$ is the susceptance of line $k$.

Also, eqn. (17) gives

$\Delta V_{p}=\sum_{u} X_{p u}^{\prime \prime} b_{i u} \Delta V_{i}$

$\Delta V_{q}=\sum_{u} X_{q u}^{\prime \prime} b_{i u} \Delta V_{i}$

where $u$ is the bus connected to bus $i$.

Substitution of eqns. (A-2) and (A-3) into (A-1) gives

$S_{J V}(k, i)=\sum_{u}\left(X_{p u}^{\prime \prime}-X_{q u}^{\prime \prime}\right) b_{i u} b_{p q}$

Derivation of $S_{V J}(j, k)$

By definition

$S_{V J}(j, k) \triangleq \frac{\Delta V_{j}}{J_{k}}$ 
where $V_{j}$ is the voltage at bus $i$ and $J_{k}$ is the base-case reactive flow in line $k$. The outage at line $k$ can be simulated with injections $\Delta Q_{p}$ and $\Delta Q_{q}$ at buses $p$ and $q$, respectively. The outage modeling criterion requires

$\Delta Q_{p}=-\Delta Q_{k}=\widehat{J}_{k}$

where the symbol refers to the condition where the outaged line is still left in the system and the injections are imposed.

The voltage changes can be calculated using eqns. (4) and (A-7):

$$
\begin{aligned}
\Delta V_{p} & =X_{p p}^{\prime \prime} \Delta Q_{p}+X_{p q}^{\prime \prime} \Delta Q_{q} \\
& =\left(X_{p p}^{\prime \prime}-X_{p q}^{\prime \prime}\right) \Delta Q_{p} \\
\Delta V_{q} & =X_{p q}^{\prime \prime} \Delta Q_{p}+X_{q q}^{\prime \prime} \Delta Q_{q} \\
& =\left(X_{p q}^{\prime \prime}-X_{q q}^{\prime \prime}\right) \Delta Q_{p} \\
\Delta V_{j} & =X_{j p}^{\prime \prime} \Delta Q_{p}+X_{j q}^{\prime \prime} \Delta Q_{q} \\
& =\left(X_{j p}^{\prime \prime}-X_{j q}^{\prime \prime}\right) \Delta Q_{p}
\end{aligned}
$$

But

$$
\begin{aligned}
\hat{J}_{k} & =b_{p q}\left(\hat{V}_{p}-\hat{V}_{q}\right) \\
& =b_{p q}\left(V_{p}-V_{q}\right)+b_{p q}\left(\Delta V_{p}-\Delta V_{q}\right) \\
& =J_{k}+b_{p q}\left(\Delta V_{p}-\Delta V_{q}\right)
\end{aligned}
$$

Substitution of eqns. (A-7), (A-8) and (A-9) into (A-12) gives

$\Delta Q_{p}=\frac{1}{1-b_{p q}\left(X_{p p}^{\prime \prime}+X_{q q}^{\prime \prime}-2 X_{p q}^{\prime \prime}\right)} J_{k}$

After combining eqns. (A-10) and (A-14),

$$
\begin{aligned}
S_{V J}(j, k) & =\frac{\Delta V_{j}}{J_{k}} \\
& =\frac{X_{j p}^{\prime \prime}-X_{j q}^{\prime \prime}}{1-b_{p q}\left(X_{p p}^{\prime \prime}+X_{q q}^{\prime \prime}-2 X_{p q}^{\prime \prime}\right)}
\end{aligned}
$$

\section{Derivation of $S_{J S}(k, i)$}

By definition

$S_{J S}(k, i) \triangleq \frac{\Delta J_{k}}{\Delta b_{i}}$

where $b_{i}$ is the shunt admittance at bus $i$.

Since

$$
\Delta b_{i}=V_{i}^{2} \Delta Q_{i} \cong \Delta Q_{i}
$$

from eqn. (4),

$$
\begin{aligned}
& \Delta V_{p}=X_{p i}^{\prime \prime} \Delta Q_{i} \\
& \Delta V_{q}=X_{q i}^{\prime \prime} \Delta Q_{i}
\end{aligned}
$$

Substitution of eqns. (A-18) and (A-19) into (A-2) gives

$S_{J S}(k, i)=\left(X_{p i}^{\prime \prime}-X_{q i}^{\prime \prime}\right) b_{p q}$

Derivation of $S_{J T}(k, l)$

By definition

$S_{J T}(k, l) \triangleq \frac{\Delta J_{k}}{\Delta t_{l}}$

where $t_{l}$ is the tap of the transformer at branch $l$.

Combining eqns. (A-2) and (23),

$S_{J T}(k, l)=\left[\left(X_{p n}^{\prime \prime}-X_{p m}^{\prime \prime}\right)-\left(X_{q n}^{\prime \prime}-X_{q m}^{\prime \prime}\right)\right] b_{p q} b_{n m}$ 